\title{
CAMA
}

Centre for Applied Macroeconomic Analysis

\section{Time-varying Trend Models for Forecasting Inflation in Australia}

\section{CAMA Working Paper 99/2020 November 2020}

Na Guo

School of Finance, Tianjin University of Finance and Economics, Tianjin

\section{Bo Zhang}

School of Economics, Shanghai University, Shanghai

Centre for Applied Macroeconomic Analysis, ANU

\section{Jamie Cross}

BI Norwegian Business School

Center of Applied Macroeconomics and Commodity Prices (CAMP)

Centre for Applied Macroeconomic Analysis, ANU

\section{Abstract}

We investigate whether a class of trend models with various error term structures can improve upon the forecast performance of commonly used time series models when forecasting CPI inflation in Australia. The main result is that trend models tend to provide more accurate point and density forecasts compared to conventional autoregressive and Phillips curve models. The best short-term forecasts come from a trend model with stochastic volatility in the transitory component, while medium to long-run forecasts are better made by specifying a moving average component. We also find that trend models can capture various dynamics in periods of significance which conventional models cannot. This includes the dramatic reduction in inflation when the RBA adopted inflation targeting, the one-off 10 per cent Goods and Services Tax inflationary episode in 2000, and the gradual decline in inflation since 2014. 


\section{Keywords}

trend model, inflation forecast, Bayesian analysis, stochastic volatility

\section{JEL Classification}

C11, C52, E31, E37

\section{Address for correspondence:}

(E) cama.admin@anu.edu.au

\section{ISSN 2206-0332}

The Centre for Applied Macroeconomic Analysis in the Crawford School of Public Policy has been established to build strong links between professional macroeconomists. It provides a forum for quality macroeconomic research and discussion of policy issues between academia, government and the private sector.

The Crawford School of Public Policy is the Australian National University's public policy school, serving and influencing Australia, Asia and the Pacific through advanced policy research, graduate and executive education, and policy impact. 


\title{
Time-varying Trend Models for Forecasting Inflation in Australia
}

\author{
Na Guo* Bo Zhang ${ }^{\dagger}$ Jamie Cross ${ }^{\ddagger}$
}

November 16, 2020

\begin{abstract}
We investigate whether a class of trend models with various error term structures can improve upon the forecast performance of commonly used time series models when forecasting CPI inflation in Australia. The main result is that trend models tend to provide more accurate point and density forecasts compared to conventional autoregressive and Phillips curve models. The best short term forecasts come from a trend model with stochastic volatility in the transitory component, while mediumto long-run forecasts are better made by specifying a moving average component. We also find that trend models can capture various dynamics in periods of significance which conventional models cannot. This includes the dramatic reduction in inflation when the RBA adopted inflation targeting, the one-off 10 per cent Goods and Services Tax inflationary episode in 2000, and the gradual decline in inflation since 2014 .
\end{abstract}

Keywords: trend model, inflation forecast, Bayesian analysis, stochastic volatility

JEL classification codes: C11, C52, E31, E37

\footnotetext{
${ }^{*}$ School of Finance, Tianjin University of Finance and Economics, Tianjin, 300222 P. R. China. Email: nkguona@gmail.com

${ }^{\dagger}$ Corresponding author: School of Economics, Shanghai University, Shanghai, 200444 P. R. China, Centre for Applied Macroeconomic Analysis (CAMA), Australian National University, Australia. Email: bozhangyc@gmail.com

$\ddagger$ BI Norwegian Business School, Center of Applied Macroeconomics and Commodity Prices (CAMP), Centre for Applied Macroeconomic Analysis (CAMA). Email: jamie.cross@bi.no
} 


\section{Introduction}

Increases in the general price level of goods and services, known as inflation, affects the decisions of households, firms and governments making it one of the most important macroeconomics indicators. Since inflation can be affected by monetary policy, a central objective of many central banks around the world is to maintain a stable rate of inflation thereby achieving stable macroeconomic outcomes. For instance, in the early 1990s, the Reserve Bank of Australia (RBA) formulated an explicit inflation target of 23 per cent, on average, over the medium term, making it one of the early adopters of the inflation targeting framework (Dixon and G.C., 2004). Since then, Australian CPI inflation has typically ranged between $0-6$ per cent in any given quarter. The Australian experience has shown that inflation is difficult to fine-tune within a narrow band. As a result, obtaining accurate inflation forecasts is critical in making correct policy decisions.

Despite being an early adopter of inflation targeting, the literature on forecasting Australian inflation is relatively sparse (see, e.g. Beechey and Österholm (2010); Garnier et al. (2015); Cross and Poon (2016) and references therein). Moreover, none of these papers has investigated whether explicit modeling of the underlying trend can enhance forecast performance. This is surprising since fluctuations in the trend, as opposed to period-to-period fluctuations, are more in line with the RBA's definition of a medium term inflation target. With this potential shortcoming in mind, our objective in this paper is to determine whether a class of trend models with various error term structures can improve upon commonly used models in the literature.

To this end, we provide the first a systematic study on forecasting Australia inflation using time-varying trend models with various specifications for flexible error structures. In trend models, the time series is decomposed into an underlying trend and transitory component. In the literature on forecasting US CPI inflation, it has been shown that allowing for time-varying volatility in both components enhances overall forecast performance (Stock and Watson, 2007). Since this is the first paper to consider such models for the Australian economy, we consider a trend model with stochastic volatility in the measurement equation (Trend-SV), a trend model with a moving average and stochastic volatility (Trend-SV-MA) (Chan, 2013), and also revisit the trend model with stochastic volatility in both the measurement equation and the state equation (Trend-2SV) (Stock and Watson, 2007). Set in this manner we can learn which trend model is the best to forecast inflation in Australia. In addition to these models, we also consider other competing models. This includes autoregressive (AR) and Phillips curve (PC) models with various specifications on the error terms, along with a combination based forecast. PC models, which rely on the unemployment rate, remain attractive models in inflation forecast (e.g., Staiger, Stock, and Watson, 1997; Brayton, Roberts, and Williams, 1999; Garratt, Mitchell, Vahey, and Wakerly, 2011). They achieved remarkable forecasting performance in some periods in the U.S. from 1984 to 1996, and remain an important candidate model for inflation forecasts in Australia.

In line with Australia's adoption of inflation targeting, our forecast evaluation sample 
ranges from 1993Q3-2019Q4. Using both point and density forecasts, our results show that trend models with stochastic volatility consistently performance well across all forecast horizons. More specifically, we find that Trend-SV forecasts well at short horizons in point forecast and all forecast horizons in density forecasts, while Trend-2SV-MA and Trend-SV-MA have the best forecasting performance on medium and long horizons in point forecasts, respectively. In the point forecast analysis, we also find that the trend models can better predict underlying changes in the inflation dynamics as compared to the AR and PC models. For instance, trend models can capture the dramatic decrease in the underlying trend of inflation when the inflation targeting policy was implemented, the a one-off 10 per cent Goods and Services Tax inflationary episode in 2000, and the gradual decline in inflation since 2014.

The remainder of the present paper proceeds as follows. Section 2 describes the specifications of the trend models. Section 3 presents the data employed in the estimation and forecasting exercises, followed by the simulation methods of the parameters. Section 4 discusses the forecast results of all the competing models for both the recursive forecast, combination forecast, and rolling window forecast. Section 5 concludes the entire paper.

\section{Trend Models and Other Competing Models}

The models used in this paper for forecasting Australia inflation can be divided into three groups. We firstly introduce the trend model group, which is time-varying trend group and specified by the underlying trend of inflation and time-varying parameters. There are then two groups of competing models: The autoregressive model (AR) group and the Phillips curve (PC) group. Since PCs are often estimated using levels or first differences, we further split the PC group into two subgroups. The first subgroup specifies PCs in terms of the inflation and the unemployment rate (PC group), and the second subgroup uses their first differences (PCd group). The following sections provide details of the specifications on error terms in each group.

\subsection{The Trend Model Group}

\subsubsection{Trend}

The first model is a trend model with a Gaussian distributed error term (Trend) and constant variances, which is defined as:

$$
\begin{aligned}
& y_{t}=\tau_{t}+\varepsilon_{t}^{y}, \quad \varepsilon_{t}^{y} \sim \mathcal{N}\left(0, \sigma_{y}^{2}\right), \\
& \tau_{t}=\tau_{t-1}+\varepsilon_{t}^{\tau}, \quad \varepsilon_{t}^{\tau} \sim \mathcal{N}\left(0, \sigma_{\tau}^{2}\right),
\end{aligned}
$$


where the error terms $\varepsilon_{t}^{y}$ and $\varepsilon_{t}^{\tau}$ are assumed to follow a Gaussian distribution, and they are serially uncorrelated and independent. Equation (1) is known as a measurement equation, in which $\tau_{t}$ is the latent trend or unobserved component. Equation (2) is the state equation which we specify as a random walk.

\subsubsection{Trend-SV}

The trend model can be extended by allowing the measurement equation to have stochastic volatility (Trend-SV):

$$
\begin{aligned}
& y_{t}=\tau_{t}+\varepsilon_{t}^{y}, \quad \varepsilon_{t}^{y} \sim \mathcal{N}\left(0, \mathrm{e}^{h t}\right), \\
& \tau_{t}=\tau_{t-1}+\varepsilon_{t}^{\tau}, \quad \varepsilon_{t}^{\tau} \sim \mathcal{N}\left(0, \sigma_{\tau}^{2}\right), \\
& h_{t}=h_{t-1}+\varepsilon_{t}^{h}, \quad \varepsilon_{t}^{h} \sim \mathcal{N}\left(0, \sigma_{h}^{2}\right),
\end{aligned}
$$

where the error terms $\varepsilon_{t}^{y}, \varepsilon_{t}^{\tau}$ and $\varepsilon_{t}^{h}$ are serially uncorrelated and independent of each other. Note that the error term in Equation (3) are specified by stochastic volatility, thereby allowing the magnitude of the variance to change over time. In contrast, Equation (4) has a constant error term.

\subsubsection{Trend-2SV}

Following Stock and Watson (2007), the Trend-SV model can be further generalized to have stochastic volatility in the state equation (Trend-2SV):

$$
\begin{aligned}
y_{t}=\tau_{t}+\varepsilon_{t}^{y}, & \varepsilon_{t}^{y} \sim \mathcal{N}\left(0, \mathrm{e}^{h t}\right), \\
\tau_{t}=\tau_{t-1}+\varepsilon_{t}^{\tau}, & \varepsilon_{t}^{\tau} \sim \mathcal{N}\left(0, \mathrm{e}^{g t}\right), \\
h_{t}=h_{t-1}+\varepsilon_{t}^{h}, & \varepsilon_{t}^{h} \sim \mathcal{N}\left(0, \sigma_{h}^{2}\right), \\
g_{t}=g_{t-1}+\varepsilon_{t}^{g}, & \varepsilon_{t}^{g} \sim \mathcal{N}\left(0, \sigma_{g}^{2}\right),
\end{aligned}
$$

where the error terms $\varepsilon_{t}^{y}, \varepsilon_{t}^{\tau}, \varepsilon_{t}^{h}$ and $\varepsilon_{t}^{g}$ are serially uncorrelated and independent of each other. 


\subsubsection{Trend-SV-MA}

Following Chan (2013), we also consider a version of the Trend-SV model in which the measurement equation has moving average errors (Trend-SV-MA):

$$
\begin{aligned}
y_{t} & =\tau_{t}+\varepsilon_{t}^{y}, \\
\tau_{t} & =\tau_{t-1}+\varepsilon_{t}^{\tau}, \quad \varepsilon_{t}^{\tau} \sim \mathcal{N}\left(0, \sigma_{\tau}^{2}\right), \\
\varepsilon_{t}^{y} & =u_{t}+\psi_{1} u_{t-1}+\cdots+\psi_{q} u_{t-q}, \quad u_{t} \sim \mathcal{N}\left(0, \mathrm{e}^{h t}\right), \\
h_{t} & =h_{t-1}+\varepsilon_{t}^{h}, \quad \varepsilon_{t}^{h} \sim \mathcal{N}\left(0, \sigma_{h}^{2}\right),
\end{aligned}
$$

where $\varepsilon_{y}^{2}$ has an MA process, and $\sigma_{\tau}^{2}$ is the variance of the underlying trend of inflation. Following Chan (2013) and Zhang et al. (2020), we set the order of moving average component to be one for simplicity.

\subsubsection{Trend-2SV-MA}

The last trend model allows for stochastic volatility in the state equation of the TrendSV-MA model (Trend-2SV-MA):

$$
\begin{aligned}
y_{t} & =\tau_{t}+\varepsilon_{t}^{y}, \\
\tau_{t} & =\tau_{t-1}+\varepsilon_{t}^{\tau}, \quad \varepsilon_{t}^{\tau} \sim \mathcal{N}\left(0, \mathrm{e}^{g t}\right), \\
\varepsilon_{t}^{y} & =u_{t}+\psi_{1} u_{t-1}+\cdots+\psi_{q} u_{t-q}, \quad u_{t} \sim \mathcal{N}\left(0, \mathrm{e}^{h t}\right), \\
h_{t} & =h_{t-1}+\varepsilon_{t}^{h}, \quad \varepsilon_{t}^{h} \sim \mathcal{N}\left(0, \sigma_{h}^{2}\right) .
\end{aligned}
$$

where the error terms $\varepsilon_{t}^{y}, \varepsilon_{t}^{\tau}, \varepsilon_{t}^{h}$ and $\varepsilon_{t}^{g}$ are serially uncorrelated and independent of each other, and $\varepsilon_{y}^{2}$ has an MA process.

\subsection{The AR Group}

In addition to trend-models we consider four AR models. This includes the standard AR with homoscedastic Gaussian distributed errors (AR), an AR with stochastic volatility (AR-SV), an AR with an MA process (AR-MA), and an AR with both stochastic volatility and an MA process (AR-SV-MA).

\subsubsection{The Benchmark AR model}

The AR model is defined as:

$$
y_{t}=\sum_{i=1}^{p} a_{i} y_{t-i}+\varepsilon_{t}, \quad \varepsilon_{t} \sim \mathcal{N}\left(0, \sigma^{2}\right) .
$$


When conducting forecasting exercise, the $k$-step ahead forecast inflation of the AR model can be calculated by

$$
y_{t+k}^{k}-y_{t}=a^{p}(A) \Delta y_{t}+\varepsilon_{t}^{k},
$$

where $a^{p}(A)$ denotes the polynomials in lag operator $A$ and $\varepsilon_{t}^{k}$ is the $k$-step ahead forecast error. Following Stock and Watson (2007), we use the Akaike Information Criterion (AIC), and also the Hannan-Quinn information criterion (HQC) to determine the values of $p$ for benchmark models in two sample periods. Based on the results of AIC and HQC, the optimal lag lengths of AR model are reported in Table A1 in the Appendix, which are lag four for the sample period 1978Q2-2019Q4, and lag one for the sub-sample 1993Q3-2019Q4 when there is inflation targeting framework.

\subsection{Phillips Curve Models}

\subsubsection{Phillips Curve I}

Following Stock and Watson $(1999,2007)$ we use a non-accelerating inflation rate of the unemployment (NAIRU) Phillips curve (PC), which is defined by:

$$
y_{t}=\sum_{i=1}^{p} b_{1 i} \Delta y_{t-i}+\sum_{j=0}^{q}\left(b_{2 j}\left(U_{t-j}-\bar{U}\right)+\varepsilon_{t}, \quad \varepsilon_{t} \sim \mathcal{N}\left(0, \sigma^{2}\right),\right.
$$

where $U$ stands for the unemployment rate and $\bar{U}$ is the natural unemployment rate. Parameters $p$ and $q$ are the lag lengths of inflation and the unemployment rate in the $\mathrm{PC}$, respectively. The $k$-step ahead forecast inflation of the $\mathrm{PC}$ is obtained as follows:

$$
y_{t+k}^{k}-y_{t}=b_{1}^{k}(B) y_{t}+b_{2}^{k}(B)\left(U_{t}-\bar{U}\right)+\varepsilon_{t}^{k},
$$

where $b_{1}^{k}(B)$ and $b_{2}^{k}(B)$ denote the polynomials in lag operator $B$, respectively. NAIRU $(\bar{U})$ is assumed to be time invariant. Let $b_{0}^{k}=-b_{2}^{k}(B) \bar{U}$; then, Equation 18 can be rewritten as Equation 19 with a constant term $b_{0}^{k}$ :

$$
y_{t+k}^{k}-y_{t}=b_{0}^{k}+b_{1}^{k}(B) \Delta y_{t}+b_{2}^{k}(B) U_{t}+\varepsilon_{t}^{k} .
$$

This Phillips curve with an unemployment rate level is a conventional Phillips curve model. The transformed Equation 19 can describe the unemployment rate directly for the inflation forecast. It is denoted as $\operatorname{PC}(p, q)$, where $p$ is the lag length of the first differential of inflation and $q$ is the lag length of the unemployment rate. As with the AR model, the lag lengths $p$ and $q$ are determined by the AIC and HQC. The results are reported in Table A1 in the Appendix, which are $p=4$ and $q=4$ in both two sample time periods, 1978Q2-2019Q4 and 1993Q3-2019Q4.

Along with PC, PC models with SV, with MA, and with SV-MA (PC-SV, PC-MA and $\mathrm{PC}-\mathrm{SV}-\mathrm{MA}$ ) are also considered as competing models in the forecasting exercises. The 
specifications of these models are the same as those in the trend model group. For simplicity, the lag lengths of inflation and the unemployment rate in these models are both four in two sample time periods to consistent with PC.

\subsubsection{Phillips Curve II}

Phillips curve II is specified by an assumption that autoregressive distributed lags (ADL) exist in both the inflation and unemployment rates, as also explored by Stock and Watson (2007). This type of Phillips curve is represented as $\operatorname{PCd}(p, q)$. The expression of $\mathrm{PCd}$ model with Gaussian distributions in error terms is below:

$$
y_{t}=\sum_{i=1}^{p} c_{1 i} y_{t-i}+\sum_{j=1}^{q} c_{2 j} \Delta U_{t-i}+\varepsilon_{t}, \quad \varepsilon_{t} \sim \mathcal{N}\left(0, \sigma^{2}\right),
$$

where both the inflation and unemployment rates are assumed to be integrated of order one I(1) and the stationary predictors $\Delta y_{t}$ and $\Delta U_{t}$ are included in the model. The $k$-step ahead forecast inflation of Phillips curve II is as follows:

$$
y_{t+k}^{k}-y_{t}=c_{1}^{k}+c_{2}^{k}(B) \Delta y_{t}+c_{3}^{k}(B) \Delta U_{t}+\varepsilon_{t}^{k},
$$

Same as AR and PC models, the lag lengths $p$ and $q$ of PCd are determined by the AIC and HQC in Table A1 in the Appendix. In 1978Q2-2019Q4, the optimal lag lengths of PCd are $p=4$ and $q=4$, while they are $p=3$ and $q=4$ in 1993Q3-2019Q4.

Same as Phillips Curve I, PCd-SV, PCd-MA and PCd-SV-MA are also introduced in the forecasting exercises. The specifications of these models are the same as corresponding trend models. For simplicity, the lag lengths of inflation and the unemployment rate in these models are consistent with PCd.

\section{Estimation}

\subsection{Data}

In the present paper, we use the quarterly consumer price index (CPI) and the unemployment rates (the percentage of the labor force 15 years and over) of Australia from 1978Q3 to 2019Q4, which are released by the Reserve Bank of Australia (RBA). Both of these macroeconomic variables are seasonally adjusted. The CPI inflation rate is calculated by the following formula:

$$
y_{t}=400 * \log \left(\mathrm{CPI}_{t} / \mathrm{CPI}_{t-1}\right) .
$$

The quarterly CPI inflation, unemployment rate, and their first differences are plotted in Figures 1 and 2. Figure 1 shows that the fluctuation in Australia inflation is stronger 
than that in the unemployment rate. Furthermore, the unemployment rate decreases gradually starting in 1993.
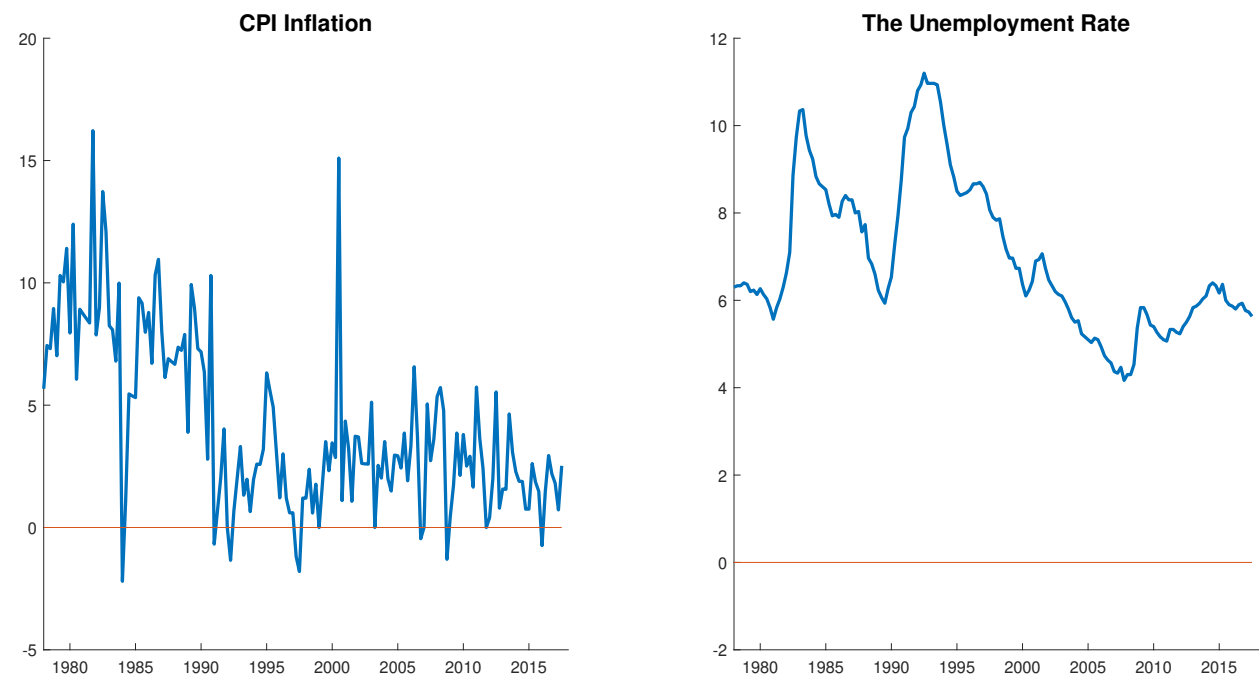

Figure 1: CPI inflation and the unemployment rates.
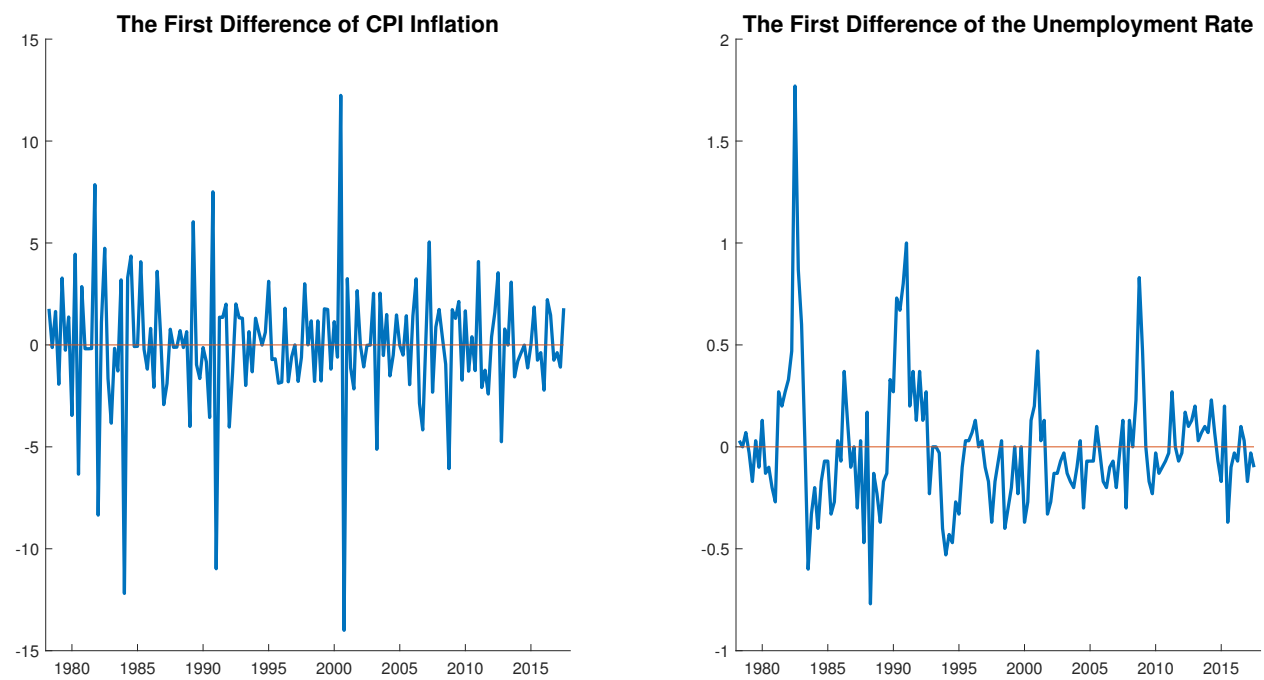

Figure 2: The first differences of CPI inflation and the unemployment rates.

\subsection{Parameter Estimation}

All models are estimated using a Bayesian paradigm with Markov chain Monte Carlo (MCMC) algorithms: Gibbs sampling and Metropolis-Hastings. Following Chan and 
Jeliazkov (2009), the latent states are sampled using an efficient precision based sampler as opposed to conventional Kalman of particle filters.

\subsection{Initial Values and Priors}

Where appropriate, the initial values of $\tau, h$ and $g$ are assumed to follow Gaussian distributions:

$$
\tau_{1} \sim \mathcal{N}\left(\tau_{0}, \sigma_{0 \tau}^{2}\right), \quad h_{1} \sim \mathcal{N}\left(h_{0}, \sigma_{0 h}^{2}\right), \quad g_{1} \sim \mathcal{N}\left(g_{0}, \sigma_{0 g}^{2}\right),
$$

where we set $\tau_{0}=h_{0}=g_{0}=0$ and $\sigma_{0 \tau}^{2}=\sigma_{0 h}^{2}=\sigma_{0 g}^{2}=5$. Therefore, the initial values of these two parameters are distributed with mean 0 and variance 5. Considering the properties of the growth rates for macroeconomic time series, the prior distribution is around 0 and large prior variances are used within $(-5,5)$, thus, the initial values are reasonable and relatively non-informative.

The priors of $\sigma_{\tau}^{2}, \sigma_{h}^{2}$, and $\sigma_{g}^{2}$ are assumed to be independent and follow inverse-gamma distributions:

$$
\sigma_{\tau}^{2} \sim \mathcal{I} \mathcal{G}\left(\nu_{\tau}, S_{\tau}\right), \quad \sigma_{h}^{2} \sim \mathcal{I} \mathcal{G}\left(\nu_{h}, S_{h}\right), \quad \sigma_{g}^{2} \sim \mathcal{I} \mathcal{G}\left(\nu_{g}, S_{g}\right)
$$

We set the hyperparameters to $\nu_{\tau}=\nu_{h}=\nu_{g}=10, S_{\tau}=0.18$, and $S_{h}=S_{g}=0.45$ based on suggestions from previous studies on inflation (e.g., Chan, 2013; Stock and Watson, 2007). These prior values imply relatively noninformative values for the shape parameters $\nu$ of the inverse-gamma distribution. The scale parameters mean that $\mathbb{E} \sigma_{\tau}^{2}=0.141^{2}$ and $\mathbb{E} \sigma_{h}^{2}=\mathbb{E} \sigma_{g}^{2}=0.224^{2}$; thus, the state transition is reasonably smooth and the results are comparable to those in the literature.

\subsection{Posterior Simulation Method}

The simulation of draws from the posterior distribution is conducted by a sequentially drawing order, and the related Bayesian inference is similar to that of Chan (2013) and Zhang (2019). Specifically, the posteriors of the trend models are sampled cyclically in the following sequence:

For the Trend model:

1. $p\left(\boldsymbol{\tau} \mid \mathbf{y}, \sigma_{y}^{2}, \sigma_{\tau}^{2}\right)$,

2. $p\left(\sigma_{y}^{2}, \sigma_{\tau}^{2} \mid \boldsymbol{\tau}\right)=p\left(\sigma_{y}^{2} \mid \boldsymbol{\tau}\right) p\left(\sigma_{\tau}^{2} \mid \boldsymbol{\tau}\right)$.

For the Trend-SV model:

1. $p\left(\boldsymbol{\tau} \mid \mathbf{y}, \mathbf{h}, \sigma_{h}^{2}, \sigma_{\tau}^{2}\right)$, 
2. $p\left(\mathbf{h} \mid \mathbf{y}, \boldsymbol{\tau}, \sigma_{h}^{2}, \sigma_{\tau}^{2}\right)$,

3. $p\left(\sigma_{h}^{2}, \sigma_{\tau}^{2} \mid \boldsymbol{\tau}, \mathbf{h}\right)=p\left(\sigma_{h}^{2} \mid \mathbf{h}\right) p\left(\sigma_{\tau}^{2} \mid \boldsymbol{\tau}\right)$.

For the Trend-2SV model:

1. $p\left(\boldsymbol{\tau} \mid \mathbf{y}, \mathbf{h}, \mathbf{g}, \sigma_{h}^{2}, \sigma_{g}^{2}\right)$,

2. $p\left(\mathbf{h}, \mathbf{g} \mid \mathbf{y}, \boldsymbol{\tau}, \sigma_{h}^{2}, \sigma_{g}^{2}\right)=p\left(\mathbf{h} \mid \mathbf{y}, \boldsymbol{\tau}, \sigma_{h}^{2}\right) p\left(\mathbf{g} \mid \mathbf{y}, \boldsymbol{\tau}, \sigma_{g}^{2}\right)$,

3. $p\left(\sigma_{h}^{2}, \sigma_{g}^{2} \mid \mathbf{h}, \mathbf{g}\right)=p\left(\sigma_{h}^{2} \mid \mathbf{h}\right) p\left(\sigma_{g}^{2} \mid \mathbf{g}\right)$.

For the Trend-SV-MA model:

1. $p\left(\boldsymbol{\tau} \mid \mathbf{y}, \mathbf{h}, \psi, \sigma_{\tau}^{2}\right)$,

2. $p\left(\mathbf{h} \mid \mathbf{y}, \boldsymbol{\tau}, \psi, \sigma_{h}^{2}\right)$,

3. $p\left(\psi, \sigma_{\tau}^{2}, \sigma_{h}^{2} \mid \mathbf{y}, \boldsymbol{\tau}, \mathbf{h}\right)=p(\psi \mid \mathbf{y}, \boldsymbol{\tau}, \mathbf{h}) p\left(\sigma_{h}^{2} \mid \mathbf{h}\right) p\left(\sigma_{\tau}^{2} \mid \boldsymbol{\tau}\right)$.

For the Trend-2SV-MA model:

1. $p\left(\boldsymbol{\tau} \mid \mathbf{y}, \mathbf{h}, \mathbf{g}, \psi, \sigma_{h}^{2}, \sigma_{g}^{2}\right)$,

2. $p\left(\mathbf{h}, \mathbf{g} \mid \mathbf{y}, \boldsymbol{\tau}, \psi, \sigma_{h}^{2}, \sigma_{g}^{2}\right)=p\left(\mathbf{h} \mid \mathbf{y}, \boldsymbol{\tau}, \psi, \sigma_{h}^{2}\right) p\left(\mathbf{g} \mid \mathbf{y}, \boldsymbol{\tau}, \psi, \sigma_{g}^{2}\right)$,

3. $p\left(\psi, \sigma_{g}^{2}, \sigma_{h}^{2} \mid \mathbf{y}, \boldsymbol{\tau}, \mathbf{h}, \mathbf{g}\right)=p(\psi \mid \mathbf{y}, \boldsymbol{\tau}, \mathbf{h}, \mathbf{g}) p\left(\sigma_{h}^{2} \mid \mathbf{h}\right) p\left(\sigma_{g}^{2} \mid \mathbf{g}\right)$.

\subsection{Posterior Estimation}

Before forecasting, we present estimates of the stochastic volatility parameters and moving average coefficient over the full sample: 1978Q2-2019Q4. All the full sample posterior estimates and quantiles are based on 50,000 draws after a burn-in with 5,000 draws.

\subsubsection{Posterior Estimates of SV Parameters}

Figure 3 presents posterior means and credible intervals of stochastic volatility parameter $h$ and $g$ obtained under Trend-SV and Trend-2SV, and Figure 4 is under Trend-SV-MA and Trend-2SV-MA. In particular, the latter models in both figures produce smoother estimates of $h$, which allow for an extra time-varying variance parameter $g$ to explain the volatility of inflation in Australia. Given that $h$ and $g$ are from exponential functions which are monotonically increasing functions, the values of $h$ and $g$ change substantially 
over the sample period, which indicates that their are substantial variation of inflation, which highlight the importance of introducing stochastic volatility in trend models.

It is also interesting to note that the curves of $h$ and $g$ vary over time. During early 1990s, the peak of $h$ under Trend-2SV-MA is lower than that under Trend-SV-MA, while the value of $g$ channeling the volatility of underlying trend from the state equation of Trend-2SV-MA also reaches a peak in that time period. However, $h$ begins to increase from 2005 under both models, while $g$ is stable around those years.
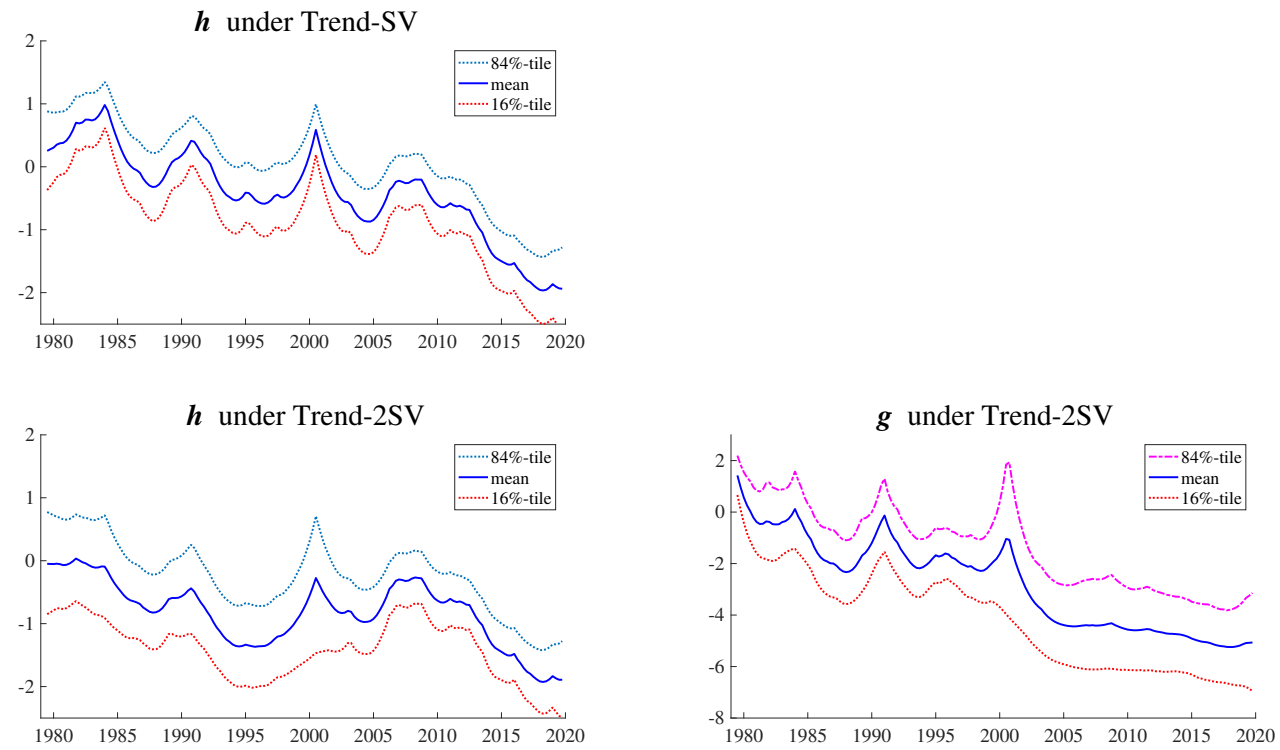

Figure 3: Posterior estimates and quantiles for SV parameter $h$ and $g$ under Trend-SV and Trend-2SV. 

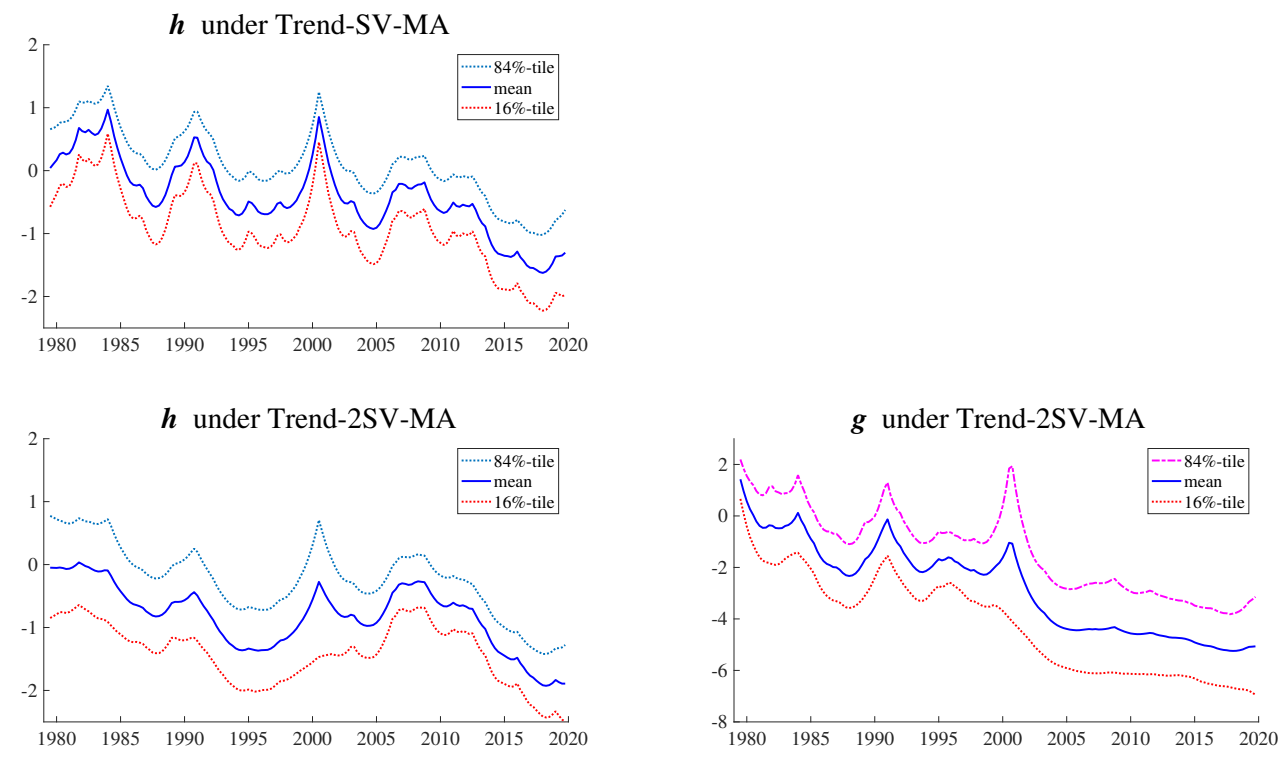

Figure 4: Posterior estimates and quantiles of SV parameter $h$ and $g$ under Trend-SV-MA and Trend-2SV-MA.

\subsubsection{Posterior distributions of MA coefficients}

The marginal density estimates for the MA coefficient $p(\psi \mid y)$ under Trend-SV-MA and Trend-2SV-MA are shown in Figure 5. Both values of $\psi$ are concentrated around 0.2 and the mass is away from 0, which suggests that MA specification is unlikely to be 0 in these two trend models. Specifically, under Trend-SV-MA, $\psi$ has higher probability and more concentrated around its mean than that under Trend-2SV-MA. It indicates that moving average component share more weight in channeling the volatility of the inflation when there is no stochastic volatility parameter in the state equation. 

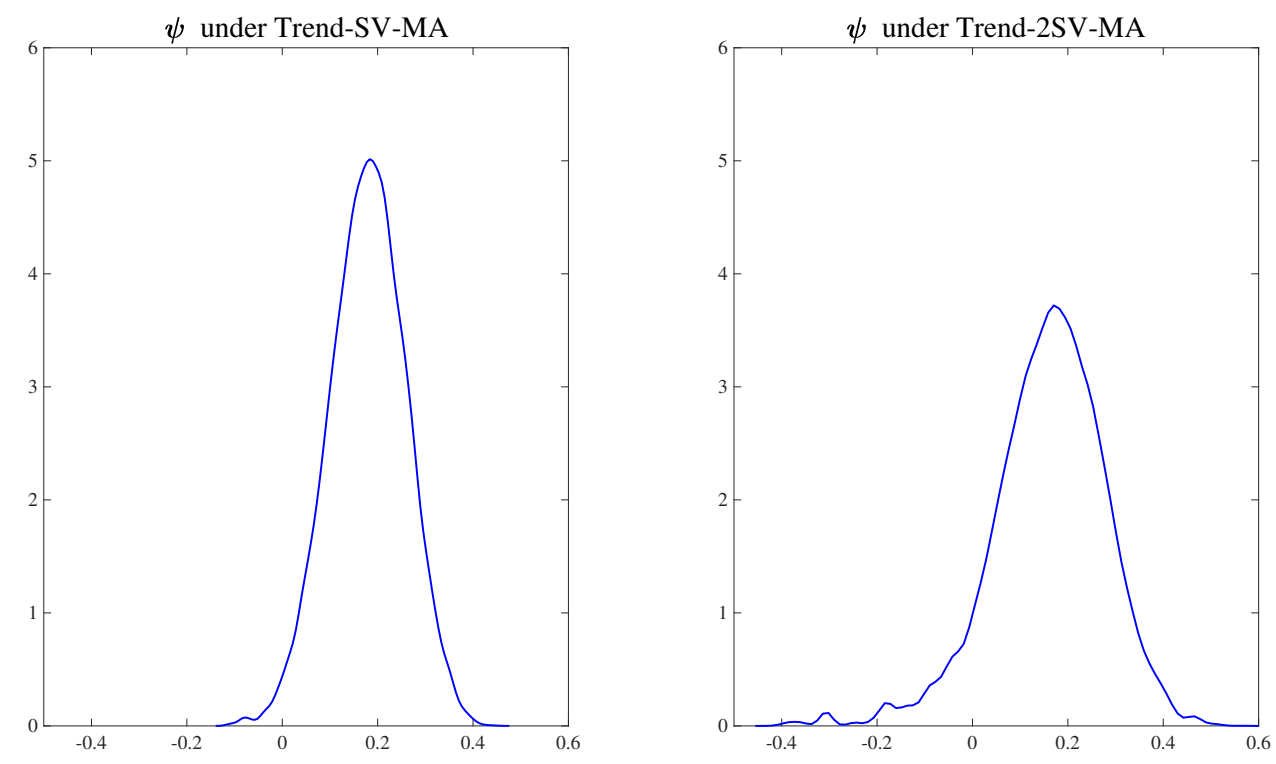

Figure 5: Posterior distribution of MA coefficients under Trend-SV-MA and Trend-2SVMA.

\section{Forecast Results}

In this section, we first discuss the forecast method used in the main analysis, including: forecast metrics, forecast horizons and sample time periods. We then present the recursive forecast results from each model, followed by combination forecast and robustness checks.

\subsection{Forecast Method}

In the forecast section, all models are estimated by a pseudo out-of-sample forecast method. That is, the forecast results are first calculated at time $T 0+1$ and compared with the actual data at time $T 0+1$. We then step to $T 0+2, T 0+3$, and so on, until we reach the end of the sample. Since quarterly time series are used, the forecast made by each model are one-quarter ahead, one-year ahead, and three-year ahead forecasts with forecast horizon $k=1,4$, and 12. Set in this manner we are able to compare short, medium and long run forecasts from each of the competing models. Since the PC group does not have an iterative formula for the unemployment rate, we use a firect forecasting methodology for all models.

The forecast performance is evaluated in terms of two metrics. Point forecasts are evaluated using the relative mean square forecast error (Relative MSFE) and density forecasts are evaluated using the relative average log predictive likelihood (Relative ALPL). 
The first forecast metric (Relative MSFE) represents a relative point forecasting performance between a candidate model and the benchmark. When calculating the MSFE of a model, the forecast $\widehat{y}_{T_{0}+t+k-1}$ is evaluated by averaging all the posterior means $\mathbb{E}\left(y_{T 0+t+k-1} \mid \mathbf{y}_{1: T_{0}+t}\right)$ at $T_{0}+t$. Then, the forecasting error is calculated by $\mathbf{e}_{T_{0}+t+k-1}^{2}=$ $\mathbf{y}_{T_{0}+t+k-1}^{0}-\mathbb{E}\left(y_{T 0+t+k-1} \mid \mathbf{y}_{1: T_{0}+t}\right)$. Eventually, the MSFE can be calculated as below:

$$
\operatorname{MSFE}=\frac{1}{T-T 0-k+1} \sum_{t=1}^{T-T 0-k+1} \mathbf{e}_{T 0+t+k-1}^{2} .
$$

For the relative MSFE, a value which is smaller than one indicates that the related candidate model has better forecasting performance than the benchmark, while a value which is larger than one means that the competing model has worse forecasting performance than the benchmark.

The predictive likelihood $p\left(\widehat{y}_{T_{0}+t+k-1}=\mathbf{y}_{T_{0}+t+k-1} \mid \mathbf{y}_{1: T_{0}+t}\right)$ is used to present the density forecasting performance $p\left(\widehat{y}_{T_{0}+t+k-1} \mid \mathbf{y}_{1: T_{0}+t}\right)$. When the observed data with higher probability fall into the density of the posterior predictive distribution, the estimated parameters which are conditional on the observed data $\mathbf{y}_{T_{0}+t+k-1}$ can produce a larger predictive likelihood value. In other words, a positive relative ALPL value suggests that the candidate model has better performance than the benchmark, while a negative ALPL value indicates that the benchmark forecasts better. The ALPL has an expression as below:

$$
\mathrm{ALPL}=\frac{1}{T-T 0-k+1} \sum_{t=1}^{T-T 0-k+1} \log p\left(\widehat{y}_{T 0+t+k-1}=y_{T 0+t+k-1} \mid \mathbf{y}_{1: T 0+t}\right)
$$

For forecast accuracy comparison, we use a one-sided sign test of equal predictive accuracy of Diebold and Mariano (1995). When the competing models are all nested, test statistics introduced by Clark and McCracken (2001) can be used. As models are allocated into four groups and are not all nested, we do not report test results of nested models. The rejection of equal forecast accuracy relative to the benchmark at significance level 0.05 is denoted by asterisk and reported in the result tables below.

The full sample time period is 1978Q2-2019Q4s. Considering that the inflation targeting framework was implemented by the RBA since early 1990s (Macfarlane, 1998), Stevens (1999) suggests that mid-1993 is the time at which the medium-term inflation target was explicitly articulated by the RBA. This time point is widely accepted as the beginning of conducting a new monetary policy, so we also report the forecasting results from sample 1993Q3-2019Q4 as a robustness check. The forecast results are estimated recursively with time marching. The first 6 points are separated for lags and the following 40 time points are used as the initial data for the parameter estimation. Thus, the forecasting periods are 1989Q4 to 2019Q4 and 2005Q1 to 2019Q4, respectively. 


\subsection{Recursive Forecast Results}

The recursive forecast results for time periods 1978Q2-2019Q4 and 1993Q3-2019Q4 are reported in Tables 1 and 2, respectively. To facilitate the discussion, we present the forecast results for two sample time periods separately. In each table, the Relative MSFE results are reported in the left column and the Relative ALPL results are in the right column.

\subsubsection{Results for Sample Period 1978Q2-2019Q4}

The forecast results in Table 1 show that trend model group perform substantially better than other model groups in both point and density forecasts (the best forecast from all the competing models in each column are in bold). When comparing trend models to the $\mathrm{AR}$ and $\mathrm{PC}$ groups, most of the forecasts from trend models perform better than the benchmark AR, which indicates that the trend group is suitable for the inflation forecast in Australia. More specifically, Trend-SV outperforms the other models on one-quarter ahead in point forecast and all the forecast horizons in density forecast, while Trend2SV-MA has the best forecasting performance on one-year ahead in point forecast and Trend-SV-MA does well on three-year forecast horizon. Trend-SV-MA also has the nearbest forecasting performance in both point and density forecasts comparing with other leading trend models. The Diebold-Mariano tests for statistical significance of point forecast improvement indicate that the gains at the long run horizon are statistically significant at 0.05 significance level. 
Table 1: Recursive forecast results for all groups, 1978Q2-2019Q4

\begin{tabular}{l|ccc|ccc}
\hline \hline & \multicolumn{3}{c}{ Relative MSFE } & \multicolumn{3}{c}{ Relative ALPL } \\
\hline & $k=1$ & $k=4$ & $k=12$ & $k=1$ & $k=4$ & $k=12$ \\
\hline AR & 1.00 & 1.00 & 1.00 & 0.00 & 0.00 & 0.00 \\
\hline AR-SV & 0.99 & 1.01 & 1.01 & 0.13 & 0.13 & 0.08 \\
AR-MA & 1.01 & 1.06 & 1.06 & -0.20 & -0.24 & -0.24 \\
AR-SV-MA & 1.00 & 1.02 & 1.02 & 0.12 & 0.12 & 0.07 \\
\hline Trend & 0.92 & 0.91 & $0.77^{*}$ & -0.03 & 0.01 & 0.12 \\
Trend-SV & $\mathbf{0 . 8 8}$ & 0.92 & $0.79^{*}$ & $\mathbf{0 . 1 4}$ & $\mathbf{0 . 1 8}$ & $\mathbf{0 . 2 6}$ \\
Trend-2SV & 1.02 & 1.03 & 0.95 & 0.13 & $0.15^{*}$ & 0.14 \\
Trend-SV-MA & 0.92 & 0.90 & $\mathbf{0 . 7 6}$ & 0.13 & 0.16 & $0.25^{*}$ \\
Trend-2SV-MA & 0.90 & $\mathbf{0 . 8 9}$ & $0.82^{*}$ & 0.05 & 0.03 & 0.06 \\
\hline PC & 1.12 & 1.24 & $2.21^{*}$ & -0.03 & -0.07 & -0.28 \\
PC-SV & 1.05 & 1.14 & $1.41^{*}$ & 0.09 & 0.05 & -0.11 \\
PC-MA & 1.12 & 1.28 & $2.48^{*}$ & -0.22 & -0.27 & $-0.40^{*}$ \\
PC-SV-MA & 1.06 & 1.16 & $1.40^{*}$ & $0.08^{*}$ & 0.05 & -0.10 \\
\hline PCd & 1.11 & 1.23 & 2.61 & -0.03 & -0.06 & $-0.28^{*}$ \\
PCd-SV & 1.04 & 1.17 & 1.66 & 0.09 & 0.04 & -0.15 \\
PCd-MA & 1.11 & 1.28 & 2.89 & -0.22 & -0.27 & $-0.41^{*}$ \\
PCd-SV-MA & 1.05 & 1.18 & 1.69 & 0.09 & 0.04 & -0.16 \\
\hline \hline
\end{tabular}

Notes: Bold entries are the smallest relative MSFE or the largest relative ALPL for the corresponding horizons. * indicates rejection of equal forecast accuracy relative to AR model at significance level 0.05, when using an asymptotic test in Diebold and Mariano (1995).

For trend models with SV parameters in both measurement equation and state equation (Trend-2SV and Trend-2SV-MA), the forecast performance of these models depends on wether MA specification is included into the model. Specifically, Trend-2SV does not have better forecasting performance than that of Trend-SV on all the forecast horizons and cannot beat other trend models in point forecast. While Trend-2SV-MA have slightly better performance than Trend-SV-MA in point forecasts, they only dominate the Trend model on short and medium run forecasts in density forecasts. Overall, Trend-SV-MA can improve both point and density forecasts which are gained by the simpler Trend model for Australia, while it is not true for a trend model with only two SV parameters.

For the AR group, the forecast results in Table 1 suggest that the benchmark AR is hard to be beat by AR models with other specifications in point forecast, while in density forecast, AR-SV and AR-SV-MA with SV specification in the measurement equation can improve forecasting performance than the benchmark across all the forecast horizons. Similarly, AR-SV-MA has optimal density forecasts on all the forecast horizons within AR group.

For PC and PCd groups, neither PC nor PCd can outperform the benchmark across all the forecast horizons and two forecast metrics. While PC and PCd models with SV and SV-MA have better forecasts than the benchmark on one-quarter and one-year ahead forecasts in density forecast, and they are also the best models within their own groups. 
In summary, the main conclusion is that the trend group tends to outperform the AR and PC groups, with particularly large improvements from models with SV and SV-MA.

\subsubsection{Point Forecast Analysis}

To give a closer examination of the performance of all the competing models, we plot the actual inflation time series and one-quarter ahead point forecasts in Figure 6. The competing models are allocated by groups to facilitate a model comparison. The main insight is that the forecasts from models in the trend group adjust rapidly with the fast jumped actual value along the evaluation period. It's also important to note that forecasts from models with SV and SV-MA specifications (especially Trend-SV and Trend-SV-MA) have less volatility and are close to the actual value. This is potentially why Trend-SV and Trend-SV-MA can outperform other models in the forecast exercise.
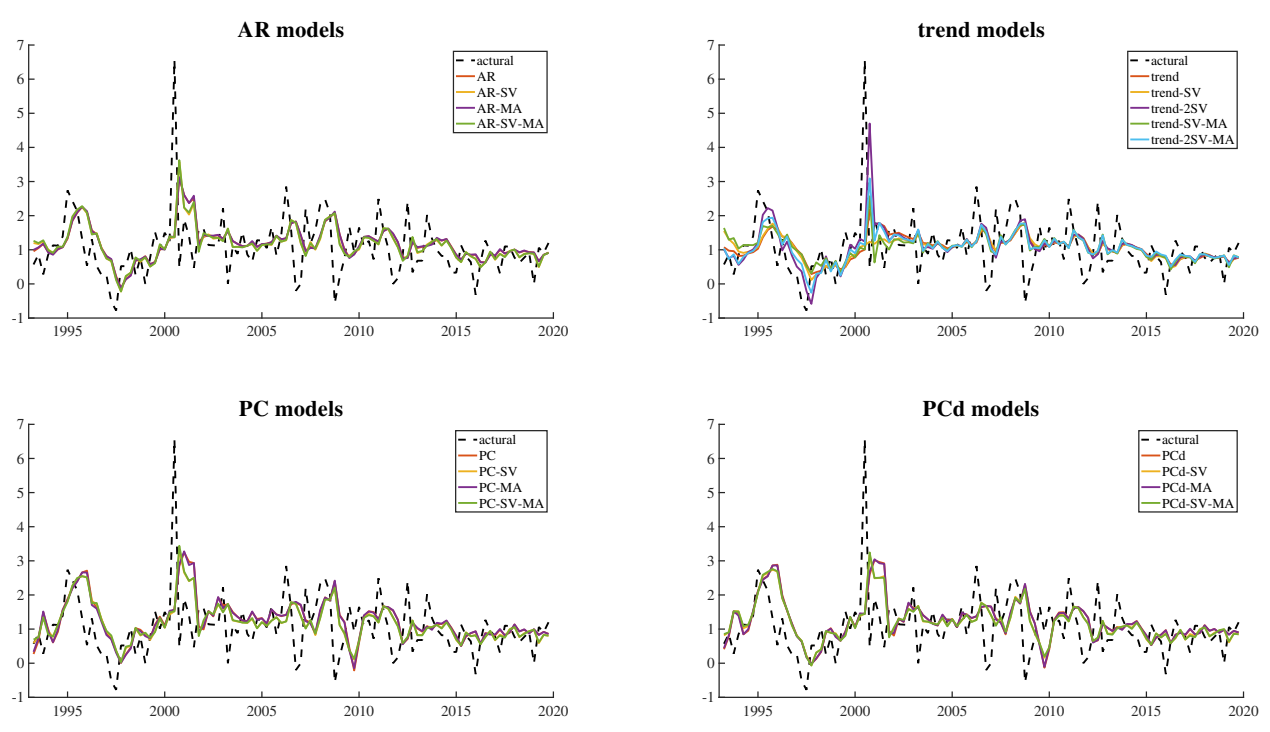

Figure 6: Actual inflation time series and one-quarter ahead point forecast under all the competing models.

We also present the MSFEs of one-quarter ahead forecasts across the entire forecasting period for each model and plot them on a recursive basis in Figure 7 . We see that the MSFEs of all the models dropped substantially around 1995, raised up dramatically in 2000, and then decreased gradually up to the date. This indicates that all the models capture the flexible specification of the inflation target in Australia during the first few years. A possible mechanism is that when the demand pressures from the real economy were eased, inflation declined, and the MSFEs raised to a platform with a sustained delayed response from model-based forecasts. 

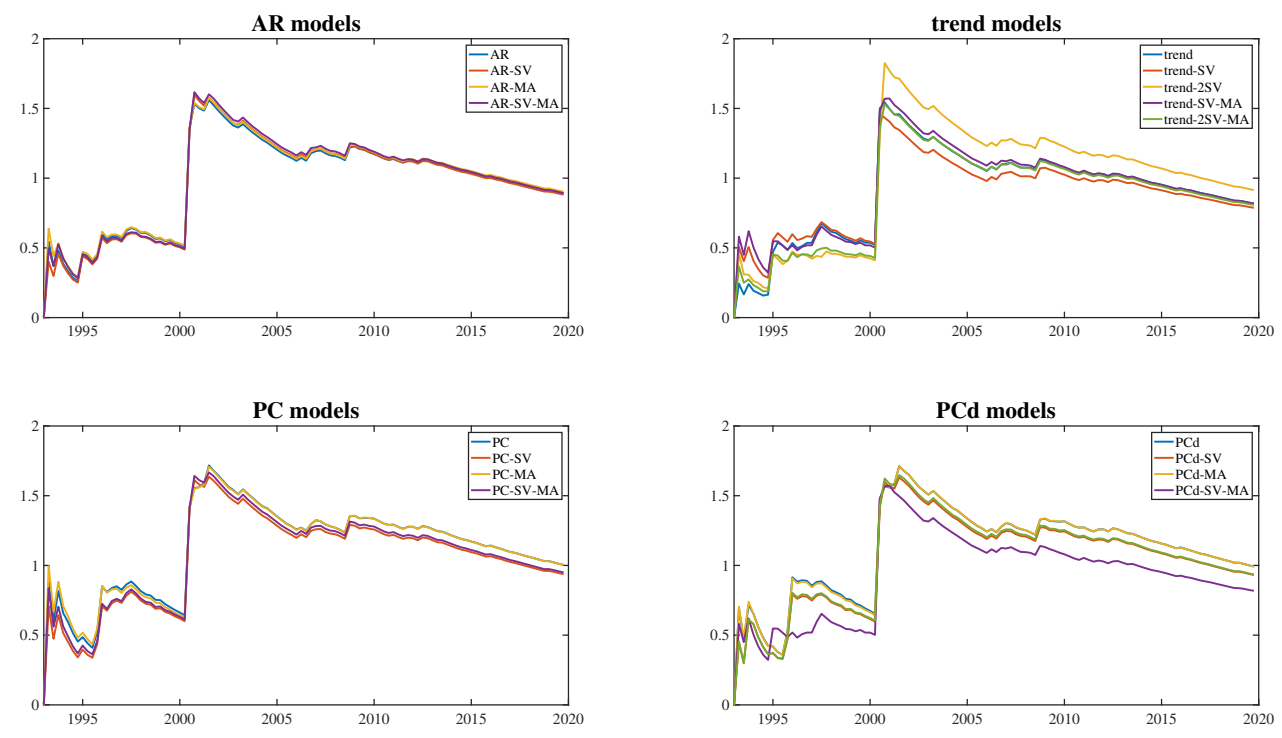

Figure 7: MSFEs of one-quarter ahead forecast under all the competing models on a recursive basis.

When the 10 per cent Goods and Services Tax was implement from 1st July, 2000, inflation immediately increased by 3 per cent overnight and shot up over 6 per cent in 2000Q3. It is a one-off boost to the price level and the inflation dropped back around 2-3 per cent inflation target in the subsequent quarters. During this inflationary episode, only trend models adjust back the underlying trend quickly, with Trend-SV having smallest MSFE of all models.

Since 2014, the Reserve Bank of Australia has undershooting the inflation target and the inflation rate is under it's lower bound of 2 per cent. The results in Figure 6 show that models with an underlying trend specification capture this feature and have smoother forecasts for inflation. The MSFEs of all the models have similar performance and all the values fall back below 1 by the end of the sample.

\subsubsection{Results for the Sample Period 1993Q3-2019Q4}

Table 2 reports the forecast results when we restrict the forecast evaluation sample to begin in 1993Q3, thereby reflecting the period in which Australia had an inflation target. We observe that the trend model group outperform other groups on medium run and long runs point forecasts. In density forecast, Trend-SV, Trend-2SV and Trend-2SV-MA have better forecasting performance than the benchmark on short and medium runs.

For the trend group, Trend-SV and Trend-SV-MA with SV in the measurement equation still outperform other trend models, suggesting the underlying trend inflation captured by Trend-SV and Trend-SV-MA accurately depicts the persistence of inflation than other 
trend models. For AR, PC and PCd groups, models with SV or SV-MA also forecast better than models with Gaussian distribution or just MA specification in most cases. Taken together, these forecast results show that models with SV or SV-MA provide good point and density forecasts for inflation in the sub-sample period.

When comparing models in the AR and PC groups, we find that only the AR-SV-MA can outperform the benchmark on one-quarter ahead point forecast and three-year ahead density forecast. Moreover, both the relative MSFE and the relative ALPL of competing models are closer to those of the benchmark than the results from the entire sample period.

Table 2: Recursive forecast results for all groups, 1993Q3-2019Q4

\begin{tabular}{l|ccc|ccc}
\hline \hline & \multicolumn{3}{c}{ Relative MSFE } & \multicolumn{3}{c}{ Relative ALPL } \\
\hline AR & $k=1$ & $k=4$ & $k=12$ & $k=1$ & $k=4$ & $k=12$ \\
\hline AR-SV & 1.00 & 1.00 & 1.00 & 0.00 & 0.00 & $\mathbf{0 . 0 0}$ \\
\hline AR-MA & 1.00 & 1.00 & 1.00 & 0.05 & 0.03 & -0.04 \\
AR-SV-MA & 1.01 & $1.03^{*}$ & 1.04 & -0.03 & $-0.05^{*}$ & -0.06 \\
\hline Trend & $\mathbf{0 . 9 9}$ & $1.00^{*}$ & 1.00 & $\mathbf{0 . 0 5}$ & 0.04 & -0.03 \\
Trend-SV & 1.11 & 0.95 & $\mathbf{0 . 9 7}$ & $-0.15^{*}$ & $-0.14^{*}$ & $-0.23^{*}$ \\
Trend-2SV & 1.11 & $\mathbf{0 . 9 4}$ & 0.98 & 0.03 & 0.07 & -0.03 \\
Trend-SV-MA & 1.17 & 0.99 & 0.99 & 0.02 & 0.05 & -0.11 \\
Trend-2SV-MA & 1.11 & $\mathbf{0 . 9 4}$ & $\mathbf{0 . 9 7}$ & 0.01 & 0.04 & -0.02 \\
\hline PC & 1.10 & 0.98 & $\mathbf{0 . 9 7}$ & $-0.06^{*}$ & $-0.07^{*}$ & $-0.18^{*}$ \\
PC-SV & 1.16 & 1.28 & $-0.12^{*}$ & -0.04 & -0.08 \\
PC-MA & 1.22 & 1.10 & 1.08 & 0.01 & $\mathbf{0 . 0 8}$ & -0.01 \\
PC-SV-MA & $1.42^{*}$ & 1.17 & 1.25 & $-0.13^{*}$ & $-0.09^{*}$ & $-0.14^{*}$ \\
\hline PCd & 1.22 & 1.11 & 1.07 & 0.01 & 0.07 & -0.01 \\
\hline PCd-SV & $1.42^{*}$ & 1.11 & 1.10 & $-0.12^{*}$ & -0.04 & -0.04 \\
PCd-MA & $1.31^{*}$ & 1.22 & 1.08 & $-0.03^{*}$ & 0.04 & -0.02 \\
PCd-SV-MA & $1.42^{*}$ & 1.10 & 1.06 & $-0.13^{*}$ & -0.09 & $-0.11^{*}$ \\
\hline \hline
\end{tabular}

Notes: See the notes to Table 1.

\subsection{Combination Forecast}

In this section, we conduct a forecasting analysis by combining the forecasting results in each group. Specifically, the forecasting results are combined by both equal weight (EW) and time-varying weight (TVW) methods for the entire sample period. Following the suggestion of Zhang (2019), the window width is forty in the time-varying weight combination forecast, that is, the time-varying weight of each model is determined by the forecasting performance of previous forty quarters. The results for both equal weight and time-varying weight forecasting results for AR group, trend group, PC and PCd groups, respectively, are shown in Table 3. 
Overall, the best forecasting performance is from time-varying weight of trend models (Trend-TVW) with optimal forecast across all the forecast horizons and methods. Moreover, equal weight of trend models (Trend-EW) has the near best forecasting performance. The close performance between equal weight and time-varying weight is also true in AR group, $\mathrm{PC}$ and $\mathrm{PCd}$ groups, and it is consistent with the conclusion that equal weight and time-varying weight have similar forecast results for the US inflation forecast in Zhang (2019). Under combination forecast, the forecasting performance of trend group has been significantly improved on long-run horizon in point forecast. The relative MSFE of Trend-EW and Trend-TVW are both 0.70 comparing to the best model Trend-SV-MA with 0.76 . Similarly to the results from recursive forecast, the improvement from trend models on the long run horizon is statistically significant in the Diebold-Mariano tests for both forecast metrics. Finally, it is worth noting that the combination forecasting performance of PC and PCd groups is greatly improved, with both their point and density forecast results being similar to those of the benchmark.

Table 3: Combination forecast results for all groups, 1978Q2-2019Q4

\begin{tabular}{l|ccc|ccc}
\hline \hline & \multicolumn{3}{c}{ Relative MSFE } & \multicolumn{3}{c}{ Relative ALPL } \\
\hline & $k=1$ & $k=4$ & $k=12$ & $k=1$ & $k=4$ & $k=12$ \\
\hline AR & 1.00 & 1.00 & 1.00 & 0.00 & 0.00 & 0.00 \\
\hline AR-EW & 1.00 & 1.00 & $0.92^{*}$ & 0.01 & 0.01 & $-0.03^{*}$ \\
AR-TVW & 1.00 & 1.00 & 0.92 & 0.02 & 0.03 & -0.01 \\
\hline Trend-EW & $\mathbf{0 . 9 1}$ & 0.89 & $\mathbf{0 . 7 0}$ & $\mathbf{0 . 1 0}$ & 0.13 & $\mathbf{0 . 1 7}^{*}$ \\
Trend-TVW & $\mathbf{0 . 9 1}$ & $\mathbf{0 . 8 8}$ & $\mathbf{0 . 7 0}$ & $\mathbf{0 . 1 0}$ & $\mathbf{0 . 1 4}$ & $\mathbf{0 . 1 7}$ \\
\hline PC-EW & 1.05 & 1.07 & 1.72 & -0.01 & -0.01 & -0.16 \\
PC-TVW & 1.05 & 1.07 & 1.80 & 0.00 & 0.00 & -0.15 \\
\hline PCd-EW & 1.04 & 1.01 & 1.54 & -0.01 & 0.00 & -0.13 \\
PCd-TVW & 1.04 & 1.01 & 1.60 & 0.00 & 0.02 & -0.11 \\
\hline \hline
\end{tabular}

Notes: See the notes to Table 1.

\subsection{Rolling Window Forecast}

As a sensitivity analysis we also conducted forecasts using a rolling window approach examine whether or not the trend model group still maintains better forecasting performance than the benchmark, the AR group and the PC groups. Unlike the recursive approach, this method fixes the parameter estimation period to a certain number of time period, in our case 40-quarters (i.e. 10 years). The Relative MSFE and ALPL metrics are reported in Tables A2 and A3 for the full sample 1978Q2-2019Q4 and restricted sample 1993Q3-2019Q4, respectively.

In the first instance, when examining the results over the full sample, we find that the relative forecast performance of the trend models to the benchmark is similar to that of the recursive forecast. However, with information from a fixed window width, no single model can improve its relative forecasting performance over information from the 
entire sample period. Among all the competing models, Trend-SV is the best forecasting model on one-quarter ahead in point forecast and almost all forecast horizons in density forecast, which is similar to the recursive forecasting results. Trend model does well in point forecast. However, both $\mathrm{PC}$ and $\mathrm{PCd}$ groups produce worse forecast than those from the benchmark across all the forecast horizons in the rolling window forecast exercise. Although PC and PCd models with SV and SV-MA outperform other models within PC and PCd groups, they are weaker than the benchmark in the rolling window forecast.

Next, when restricting the sample to the inflation targeting period, we find that all the trend models perform well on medium and long runs in point forecast. All models in PC and PCd groups outperform the benchmark on one-year ahead point forecast in rolling window forecast, however the benchmark is hard to beat in density forecast by most competing models.

In summary, the forecast results of the rolling window forecast method indicate that the trend model group is less influenced by the change of information available for estimation. Generally, trend models can provide better forecast performance than other groups. Models with SV and SV-MA specifications have better forecasting performance than those without them, which are the same as the findings in recursive forecast.

\section{Concluding Remarks}

In this paper, we compared the forecast accuracy of trend models against commonly used autoregressive and Phillips curve models when forecasting the CPI inflation rate in Australia. The results showed that a trend model with stochastic volatility in the transitory component (Trend-SV) provides the best point forecast performance at a short horizonand at all horizons in density forecast. Allowing for moving average (Trend-SV-MA and Trend-2SV-MA) further improved point forecast accuracy at both medium- and long-run horizons. This result suggests that policy makers would benefit from adopting trend models when forecasting inflation. Notably, trend models produced highly competitive forecasts when there was an inflationary episode or a permanently lower trend growth period. More generally, we also found that trend models can capture various dynamics in periods of significance which the AR and PC models cannot. This includes the dramatic reduction in inflation when the RBA adopted inflation targeting, the a one-off 10 per cent Goods and Services Tax inflationary episode in 2000, and the gradual decline in inflation since 2014. 


\section{Appendix}

Table A1: Akaike Information Criterion (AIC) and Hannan-Quinn Information Criterion (HQC) for $\operatorname{AR}(p)$ Models and $\operatorname{PC}(p, q)$ and $\operatorname{PCd}(p, q)$ Models.

\begin{tabular}{lcc|cc}
\hline \hline & \multicolumn{2}{c}{ 1978Q3-2019Q4 } & \multicolumn{2}{c}{ 1993Q3-2019Q4 } \\
\hline & AIC & HQC & AIC & HQC \\
\hline AR & 4 & 4 & 1 & 1 \\
PC & $(4,4)$ & $(4,4)$ & $(4,4)$ & $(4,4)$ \\
PCd & $(4,4)$ & $(4,4)$ & $(3,4)$ & $(3,4)$ \\
\hline \hline
\end{tabular}

Table A2: Rolling window forecast results for all groups, 1978Q2-2019Q4

\begin{tabular}{l|ccc|ccc}
\hline \hline & \multicolumn{3}{c}{ Relative MSFE } & \multicolumn{3}{c}{ Relative ALPL } \\
\hline & $k=1$ & $k=4$ & $k=12$ & $k=1$ & $k=4$ & $k=12$ \\
\hline AR & 1.00 & 1.00 & 1.00 & 0.00 & 0.00 & 0.00 \\
\hline AR-SV & 1.02 & 1.00 & 1.02 & $\mathbf{0 . 0 4}$ & -0.01 & -0.08 \\
AR-MA & 1.01 & 1.11 & 1.21 & -0.11 & -0.10 & -0.10 \\
AR-SV-MA & 1.05 & 1.01 & 1.03 & 0.03 & -0.02 & -0.08 \\
\hline Trend & 0.96 & $\mathbf{0 . 9 8}$ & $\mathbf{0 . 8 0}$ & -0.14 & -0.15 & -0.12 \\
Trend-SV & $\mathbf{0 . 9 3}$ & 1.01 & 0.83 & 0.03 & $\mathbf{0 . 0 1}$ & $\mathbf{0 . 0 2}$ \\
Trend-2SV & 1.07 & 1.11 & 1.01 & 0.00 & -0.06 & -0.17 \\
Trend-SV-MA & 1.02 & 1.01 & 0.81 & 0.00 & 0.00 & 0.04 \\
Trend-2SV-MA & 0.99 & 0.99 & $0.87^{*}$ & -0.07 & -0.16 & -0.20 \\
\hline PC & 1.16 & 1.24 & 2.49 & -0.06 & -0.09 & -0.29 \\
PC-SV & 1.13 & 1.13 & 1.53 & -0.02 & -0.08 & -0.26 \\
PC-MA & $1.17^{*}$ & 1.38 & $3.50^{*}$ & $-0.16^{*}$ & -0.15 & -0.28 \\
PC-SV-MA & $1.12^{*}$ & 1.10 & 1.39 & 0.00 & -0.06 & -0.20 \\
\hline PCd & $1.21^{*}$ & 1.26 & 2.59 & -0.08 & -0.10 & -0.36 \\
PCd-SV & $1.13^{*}$ & 1.13 & 1.53 & -0.03 & -0.08 & -0.26 \\
PCd-MA & $1.21^{*}$ & 1.36 & 3.33 & -0.19 & -0.15 & -0.29 \\
PCd-SV-MA & $1.21^{*}$ & 1.13 & 1.52 & -0.04 & -0.08 & -0.25 \\
\hline \hline
\end{tabular}

Notes: See the notes to Table 1. 
Table A3: Rolling window forecast results for all groups, 1993Q3-2019Q4

\begin{tabular}{l|ccc|ccc}
\hline \hline & \multicolumn{3}{c}{ Relative MSFE } & \multicolumn{3}{c}{ Relative ALPL } \\
\hline & $k=1$ & $k=4$ & $k=12$ & $k=1$ & $k=4$ & $k=12$ \\
\hline AR & $\mathbf{1 . 0 0}$ & 1.00 & 1.00 & $\mathbf{0 . 0 0}$ & 0.00 & $\mathbf{0 . 0 0}$ \\
\hline AR-SV & $\mathbf{1 . 0 0}$ & 1.00 & 1.00 & $\mathbf{0 . 0 0}$ & -0.04 & -0.10 \\
AR-MA & 1.01 & 0.99 & 1.00 & -0.03 & -0.04 & -0.06 \\
AR-SV-MA & 1.05 & 1.00 & 1.00 & -0.01 & -0.04 & -0.11 \\
\hline Trend & 1.05 & 0.95 & 0.91 & $-0.24^{*}$ & $-0.25^{*}$ & $-0.30^{*}$ \\
Trend-SV & 1.06 & 0.94 & 0.92 & -0.03 & 0.00 & -0.07 \\
Trend-2SV & 1.13 & 0.99 & $\mathbf{0 . 8 9}$ & -0.07 & -0.09 & $-0.24^{*}$ \\
Trend-SV-MA & 1.13 & 0.96 & 0.92 & -0.09 & -0.06 & $-0.08^{*}$ \\
Trend-2SV-MA & 1.16 & 0.98 & 0.90 & $-0.18^{*}$ & $-0.22^{*}$ & $-0.32^{*}$ \\
\hline PC & 1.25 & 0.95 & 1.28 & -0.06 & 0.00 & -0.13 \\
PC-SV & 1.10 & 0.94 & 0.98 & -0.02 & $\mathbf{0 . 0 1}$ & -0.08 \\
PC-MA & 1.25 & 0.95 & 1.25 & -0.11 & -0.10 & $-0.22^{*}$ \\
PC-SV-MA & 1.11 & 0.93 & 0.98 & -0.03 & $\mathbf{0 . 0 1}$ & -0.07 \\
\hline PCd & 1.33 & 0.94 & $1.22^{*}$ & -0.11 & -0.02 & $-0.11^{*}$ \\
PCd-SV & 1.17 & 0.93 & 1.08 & -0.08 & 0.00 & -0.11 \\
PCd-MA & 1.31 & $\mathbf{0 . 9 0}$ & 1.22 & -0.13 & -0.09 & $-0.19^{*}$ \\
PCd-SV-MA & 1.15 & 0.92 & 1.06 & -0.07 & 0.00 & -0.10 \\
\hline \hline
\end{tabular}

Notes: See the notes to Table 1. 


\section{References}

M. Beechey and P. Österholm. Forecasting inflation in an inflation-targeting regime: A role for informative steady-state priors. International Journal of Forecasting, 26(2): 248-264, 2010.

F. Brayton, J. M. Roberts, and J. C. Williams. What happened to the Phillips curve? FEDS Working Paper, 1999.

J.C.C. Chan. Moving average stochastic volatility models with application to inflation forecast. Journal of Econometrics, 176(2):162-172, 2013.

J.C.C. Chan and I. Jeliazkov. Efficient simulation and integrated likelihood estimation in state space models. International Journal of Mathematical Modelling and Numerical Optimisation, 1:101-120, 2009.

T.E. Clark and M.W. McCracken. Tests of equal forecast accuracy and encompassing for nested models. Journal of Econometrics, 105(1):85-110, 2001.

J. Cross and A. Poon. Forecasting structural change and fat-tailed events in Australian macroeconomic variables. Economic Modelling, 58:34-51, 2016.

F.X. Diebold and R.S. Mariano. Comparing predictive accuracy. Journal of Business and Econometric Statistics, 13(3):134-144, 1995.

R. Dixon and Lim G.C. Underlying inflation in Australia: Are the existing measures satisfactory. Economic Record, 80(251):373-386, 2004.

C. Garnier, E. Mertens, and E. Nelson. Trend inflation in advanced economies. International Journal of Central Banking, 11(4):5-136, 2015.

A. Garratt, J. Mitchell, S. Vahey, and E. Wakerly. Real-time inflation forecast densities from ensemble Phillips curves. The North American Journal of Economics and Finance, 22(1):77-87, 2011.

I.J. Macfarlane. Australian monetary policy in the last quarter of the twentieth century. Reserve Bank of Australia Bulletin, 10:6-19, 1998.

D. Staiger, J. Stock, and M. Watson. The NAIRU, unemployment and monetary policy. Journal of Economic Perspectives, 11(1):33-49, 1997.

G. Stevens. Six years of inflation targeting. Reserve Bank of Australia Bulletin, 5:46-61, 1999.

J.H. Stock and M.W. Watson. Forecasting inflation. Journal of Monetary Economics, 44 (2):293-335, 1999.

J.H. Stock and M.W. Watson. Why has U. S. inflation become harder to forecast. Journal of Money Credit and Banking, 39(1):3-33, 2007. 
B. Zhang. Real-time inflation forecast combination for time-varying coefficient models. Journal of Forecasting, 38(3):175-191, 2019.

B. Zhang, J.C.C. Chan, and J.L. Cross. Stochastic volatility models with ARMA innovations: An application to G7 inflation forecasts. Internatianal Journal of Forecasting, 36(4):1318-1328, 2020. 